\title{
GMR
}

\section{Fine mapping and characterization of the or gene in Chinese cabbage (Brassica rapa L. ssp pekinensis)}

C.L. Zou ${ }^{1,2 *}$, Y. Zheng ${ }^{1 *}$, P. Wang ${ }^{2}$, X. Zhang ${ }^{2}$, Y.-H. Wang ${ }^{1}$, Z.Y. Liu ${ }^{1}$ and H. Feng ${ }^{1}$

${ }^{1}$ Department of Horticulture, Shenyang Agricultural University, Shenyang, China

${ }^{2}$ Liaoning Academy of Agricultural Sciences, Shenyang, China

*These authors contributed equally to this study.

Corresponding authors: H. Feng / Z.Y. Liu

E-mail: fenghuiaaa@263.net/78160708@qq.com

Genet. Mol. Res. 15 (2): gmr. 15028370

Received December 29, 2015

Accepted February 11, 2016

Published April 27, 2016

DOI http://dx.doi.org/10.4238/gmr.15028370

\begin{abstract}
Orange inner leaves/heads is a qualitative trait in Chinese cabbage that is controlled by a single recessive gene. Orange head Chinese cabbage contain more carotenoids than its white head counterpart; hence, this trait is of interest to both researchers and consumers. In this study, we selected the orange head Chinese cabbage line 07A163 and the white head Chinese cabbage line Chiifu as test materials. We localized the target gene controlling the orange head trait to the A09 linkage group, with a physical distance of approximately $19.9 \mathrm{~kb}$ between the two markers, syau 26 and syau28. This region contains six candidate genes, including Bra031539, which was predicted to encode CRTISO, a carotenoid isomerase specifically required for carotenoid biosynthesis. A comparison of the nucleic acid sequences of the two test materials revealed 88 and 7-bp deletions and 88 SNPs in the promoter region of Bra031539 in line 07A163, along with a 6-bp deletion in the first exon and early termination at the 3 ' end
\end{abstract}


of this gene. BLAST analysis revealed that 22 amino acids were altered and 17 amino acids were lost in Bra031539 in the orange head line 07A163. We developed the BrProl molecular marker in the promoter region of Bra031539 that can be used for early identification of orange head materials, thereby accelerating the breeding process of orange head Chinese cabbage.

Key words: Chinese cabbage; Fine mapping; CRTISO;

Molecular marker; Orange head trait; Sequence blasting

\section{INTRODUCTION}

In recent years, orange head Chinese cabbage has become a popular focus of study for vegetable breeders. The inner leaves of Chinese cabbage are usually white or yellow and rarely orange. Much research has focused on the orange inner leaf trait in this important crop. More carotenoids accumulate in orange head Chinese cabbage than in white head Chinese cabbage (Watanabe et al., 2011).

An important role of carotenoids in plants is providing flowers and fruits with distinctive colors. The carotenoid biosynthetic pathway begins with the production of two 20-carbon geranylgeranyl diphosphate (GGPP) molecules that are catalyzed by geranylgeranyl diphosphate synthase (GGPS). Two GGPPs then form 15-cis phytoene via phytoene synthase (PSY), the first rate-limiting enzyme in the carotenoid biosynthesis pathway (Bramley et al., 1992; Fraser et al., 1994). Next, 15-cis phytoene is converted to tetra-cis-lycopene by two phylogenetically related enzymes: phytoene desaturase (PDS) and $\zeta$-carotene desaturase (ZDS) (Nisar et al., 2015). Carotenoid isomerase (CRTISO) and $\zeta$-carotene isomerase (Z-ISO) function as carotenoid isomerases in plants during the conversion of tetra-cis-lycopene to all-trans-lycopene (Li et al., 2007; Chen et al., 2010). The cyclization of lycopene is an important step in carotenoid metabolism; the substrate for cyclases is all-trans-lycopene. There are two branches in this step: $\alpha$-carotene is formed via lycopene $\varepsilon$-cyclase ( $\varepsilon$-LCY), followed by lycopene $\beta$-cyclase ( $\beta$-LCY), whereas $\beta$-carotene is formed via $\beta$-LCY only (Cunningham et al., 1993; Cunningham et al., 1996; Pecker et al., 1996; Ronen et al., 1999). Using these carotenes, a series of enzymes catalyze the production of abscisic acid, strigolactones, carlactone, $\beta$-citraurin, volatiles, and scents. Much progress has been made in elucidating the carotenoid biosynthesis mechanism in Arabidopsis (RuizSola and Rodríguez- Concepción, 2012) and tomato (Fraser et al., 1994), but less is known about this process in other, non-model plants.

The abnormal accumulation of carotene in the orange head Chinese cabbage is controlled by a single recessive gene (Feng et al., 2012). To analyze the reason behind this trait in Chinese cabbage, extensive research has been carried out to investigate the composition of carotenoids, develop molecular markers, complete fine mapping of the target gene and so on. The total carotenoid content in orange head Chinese cabbage is much higher than that in its white head counterpart. Moreover, lycopene is only present in orange head Chinese cabbage (Qing and Cheng, 2000; Chen, 2008; Watanabe et al., 2011; Tuan et al., 2011). Previous studies have led to the development of many molecular markers, such as SSR markers (Feng et al., 2012; Zhang et al., 2013), SCAR markers (Zhang et al., 2008), RAPD markers and so on, which are located near the or gene on linkage group A09. Bra031539 is one such gene that is 
in the target region considered to harbor the predicted candidate gene that encodes carotenoid isomerase (Zhang et al., 2013). In the current study, we analyzed the nucleic acid sequence structure and protein sequence characteristics of this target gene utilizing fine mapping. A comparison of wild-type Chinese cabbage (white head) and orange head Chinese cabbage revealed some differences between these materials. We developed a gene-specific marker that can be used to accelerate the breeding process of orange head Chinese cabbage.

\section{MATERIAL AND METHODS}

\section{Plant materials and mapping population}

The B. rapa inbred lines 07A163 and Chiifu were used as materials in the present study. Line 07A163 (which has orange inner leaves) and Chiifu (which has white inner leaves) were provided by Shenyang Agricultural University, China. We previously established a large F2 population of 2,200 individuals with the recessive orange-colored inner leaf trait from the two parents and localized the or gene to a target region between BAC clones KBrH010M08 and KBrB017P15 on chromosome A09 (Feng et al., 2012).

Germinated seeds of the $\mathrm{F} 2$ population were vernalized at $4^{\circ} \mathrm{C}$ for approximately 20 days and sown in a seedling plate. All plants were grown in a solar greenhouse under natural conditions. After flowering, recessive individuals in the fine mapping population were identified based on the presence of orange petals.

\section{PCR amplification and SSR marker development}

Genomic DNA was extracted from fresh leaves of the parents and F2 individuals as described by Murray and Thompson (1980) with minor modifications. The concentration of genomic DNA was estimated by examination on agarose gels and adjusted to $50 \mathrm{ng} / \mu \mathrm{L}$.

Polymerase chain reaction (PCR) was carried out in a volume of $10 \mu \mathrm{L}$ : each reaction contained 1.0 $\mu \mathrm{L}$ 10X Taq Reaction Buffer (containing $\mathrm{Mg}^{2+}$ ), $1 \mathrm{U}$ Taq DNA Polymerase, $0.8 \mu \mathrm{L}$ of $2.5 \mathrm{mM}$ dNTP, $1 \mu \mathrm{L}$ of $0.5 \mu \mathrm{M}$ primer and $50 \mathrm{ng}$ template DNA. The PCR program used on the Bio-Rad (USA) iCycler Thermal Cycler was as follows: an initial denaturation at $94^{\circ} \mathrm{C}$ for 5 min, followed by 30 cycles at $94^{\circ} \mathrm{C}$ for $30 \mathrm{~s}, 57^{\circ} \mathrm{C}$ for $30 \mathrm{~s}$ and $72^{\circ} \mathrm{C}$ for $30 \mathrm{~s}$, with a final extension at $72^{\circ} \mathrm{C}$ for $10 \mathrm{~min}$. The PCR products were separated on a $6 \%$ denaturing polyacrylamide gel in 1X TBE buffer, and the gel was stained with $2 \mathrm{~g} / \mathrm{L}$ silver nitrate after electrophoresis.

Two SSR markers, syau19 and syau15, were used to screen 2,200 F2 recessive individuals. After confirming the target region containing the candidate or gene, the program Primer Premier 5.0 was used to design primers for markers more closely linked to the or gene candidate. Several new markers were designed to reduce the distance from the candidate gene using the 2,200 F2 recessive individuals to calculate the recombination frequency for fine mapping. Linkage relationships were determined with DNAMAN 5.2, and the map distances were calculated in $\mathrm{cM}$.

\section{Candidate gene prediction and gene cloning}

Gene sequences of B. rapa deposited in the Brassica database (http://brassicadb. org/brad/index.php) were aligned using the BLAST program from the National Center for 
Biotechnology Information (http://blast.ncbi.nlm.nih.gov/Blast.cgi). All genes located within the interval covering the target gene were identified and the candidate gene was predicted to be Bra031539.

Based on the Bra031539 nucleotide sequence, 11 pairs of PCR primers were designed for cloning of the full-length gene (Table 1). PCR products from both parental lines were purified using a PCR purification kit for TA cloning and sequenced by SinoGenoMax Co., Ltd. (Beijing, China). The sequences were analyzed using ClustalX1.83 software.

\section{Analysis of the protein sequences of Bra031539}

DNAStar software was used to deduce the amino acid sequence of Bra031539, its subcellular localization was predicted using TargetP (http://www.cbs.dtu.dk/services/TargetP), SignalP (http://www.cbs.dtu.dk/services/SignalP) was used to predict the signal peptide region, the modification sites were identified using the Prosite program (http://www.expasy. $\mathrm{ch} /$ prosite), and the predicted product of Bra031539 was characterized via comparison to the conserved domain database using the SMART program (http://smart.embl-heidelberg.de).

\section{Development of the molecular marker based on differences in the promoter region}

Three primers were designed for the promoter region of Bra031539 based on the differences in nucleotide sequences between Chiifu and 07A163 (Table 2). White, yellow and orange head Chinese cabbage were used to verify these primers. F1 populations were established by crossing the orange line 07A163 with white lines (Chiifu, 14A014 and 14A065) and yellow lines (14A036, 14A038 and 14A029), respectively. Members of the F2 segregating population were used to verify the markers.

Table 1. Eleven primers used for Bra031539 sequence amplification.

\begin{tabular}{l|l|l|c}
\hline Primers & Forward $\left(5^{\prime}-3^{\prime}\right)$ & Reverse $\left(5^{\prime}-3^{\prime}\right)$ & Product length \\
\hline $\mathrm{Br} 1$ & GATTAGGATTTAGGGTTTAGTG & GATTCTTCTTCCGATTCTTT & 750 \\
\hline $\mathrm{Br} 2$ & GCCCAACGGATAGGTGTAAC & CATCTCACGACCAACTGCTT & 1064 \\
\hline $\mathrm{Br} 3$ & AGCGATAAGGTTCGTTTCAT & TCCATAGTGCCTGTCACATA & 1073 \\
\hline $\mathrm{Br} 4$ & AGCGATAAGGTTCGTTTCAT & TACAGCCTTTCCATCATCAA & 1205 \\
\hline $\mathrm{Br} 5$ & GCTCGGAAGTACATAAAGGA & TTGGGATGCTGAGGAAGATA & 1124 \\
\hline $\mathrm{Br} 6$ & CCCTTTCTGTTCAGTTTCTA & CCTCTTACCTCCCAATCTTC & 990 \\
\hline $\mathrm{Br} 7$ & TACAGATTGCCATCATTTCG & AGCCACAGCTATAACTCCCT & 778 \\
\hline $\mathrm{Br} 8$ & TACAGATTGCCATCATTTCG & GATTAGATTCCTTGCCCATT & 1205 \\
\hline $\mathrm{Br} 9$ & CTATGTTCCTGCCTAGATTT & GTTGAGATTAGATTCCTTGC & 742 \\
\hline $\mathrm{Br} 10$ & CTATGTTCCTGCCTAGATTT & GATGATACATGAACCAAACT & 796 \\
\hline $\mathrm{Br} 11$ & CACAGTGACGACGAACCAAG & TCTCACGACCAACTGCTTTC & 75 \\
\hline
\end{tabular}

Table 2. Molecular marker primers.

\begin{tabular}{l|l|l|c}
\hline Primers & Forward $\left(5^{\prime}-3^{\prime}\right)$ & Reverse $\left(5^{\prime}-3^{\prime}\right)$ & Product length \\
\hline BR1 & TAGATTTTATGAATACGGGACA & CGAATTTGGAAAATTGGTTT & 642 \\
\hline BR2 & TTATGAATACGGGACATG & TTGGATAGTACCAAACCT & 700 \\
\hline BR3 & TTATGAATACGGGACATG & ACACTCAGAAGTGCAGGA & 578 \\
\hline
\end{tabular}




\section{RESULTS}

\section{Fine mapping of the or gene and candidate gene analysis}

In the 2,200 F2 recessive individuals used to fine map the or gene, recombination occurred between SSR markers syau 19 and syau 15 and the target gene in 77 and 115 individuals, respectively. To obtain markers more closely linked to the target gene, we designed five SSR markers in the interval between syau19 and syau15 (Table 3). SSR markers syau 26 and syau 28 were ultimately localized to a $19.9 \mathrm{~kb}$ region, with a mapping distance between the markers and the candidate gene of $0.11 \mathrm{cM}$ and $0.09 \mathrm{cM}$, respectively (Figure 1).

DNA sequence analysis of the 19.9-kb region based on the Brassica database (http:// brassicadb.org/brad/index.php) revealed that it contains six genes (Table 4). Of these genes, Bra031539 encodes the carotenoid isomerase CRTISO, an isomerase enzyme specific to the carotenoid biosynthetic pathway.

Table 3. Sequences, number of recombinants and map distances of SSR marker primers used for fine mapping.

\begin{tabular}{l|l|l|c|c}
\hline Markers & Forward $\left(5^{\prime}-3^{\prime}\right)$ & Reverse $\left(5^{\prime}-3^{\prime}\right)$ & Recombinant & Map distance $(\mathrm{cM})$ \\
\hline syau 19 & CCGAAGCCCAGATTAGGAG & GCCCATGCCTACGAATGATAT & 77 & 1.75 \\
\hline syau 4 & TTTAAGGACTAATTAACGAGGTTTAAT & ACGGAGTGATTTATTTACATG & 12 & 0.27 \\
\hline syau 26 & TTTTGGTTTCACTGATGTTTCTT & TCCAAGTTCTTCCTTTCCTATTT & 5 & 0.11 \\
\hline syau 28 & ATCATCCACTGGTCTTATCCTCC & AGTTTACAGAGCGAACGAAGC & 4 & 0.09 \\
\hline syau 30 & TGAATTACGATGTATCTAACTGGTT & GATTGTAAAGATTCTACGTCAAAGG & 33 & 0.75 \\
\hline syau 15 & TCAAAATGCTTACAACTTCAATCTC & CATCAAGCGTATCATTTCCTGT & 34 & 0.77 \\
\hline
\end{tabular}

Table 4. Summary of predicted genes in the genomic target region on B. rapa LG A09.

\begin{tabular}{l|c|c|l|c}
\hline Gene ID & Start & End & Best hits in GenBank [accession/species] & E value \\
\hline Bra031536 & 37686812 & 37689589 & $\begin{array}{l}\text { P-loop containing nucleoside triphosphate hydrolases superfamily protein } \\
{[\text { NP_001184924/Arabidopsis thaliana] }}\end{array}$ & $0.00 \mathrm{E}+00$ \\
\hline Bra031537 & 37681637 & 37684412 & Probable galacturonosyltransferase 6 [NP_563771/ Arabidopsis thaliana] & $0.00 \mathrm{E}+00$ \\
\hline Bra031538 & 37680010 & 37681151 & $\begin{array}{l}\text { RNA polymerase Rpb7 N-terminal domain-containing protein [NP_172164/Arabidopsis } \\
\text { thaliana] }\end{array}$ & $2.00 \mathrm{E}-105$ \\
\hline Bra031539 & 37676627 & 37679733 & Carotenoid isomerase [NP_172167/Arabidopsis thaliana] & $0.00 \mathrm{E}+00$ \\
\hline Bra031540 & 37674379 & 37674678 & Glutaredoxin family protein [XP_002889620/Arabidopsis lyrata subsp. lyrata] & $3.00 \mathrm{E}-62$ \\
\hline Bra031541 & 37671570 & 37673430 & Similar to bZIP transcription factors [AAF63137/Arabidopsis thaliana] & $4.00 \mathrm{E}-142$ \\
\hline
\end{tabular}

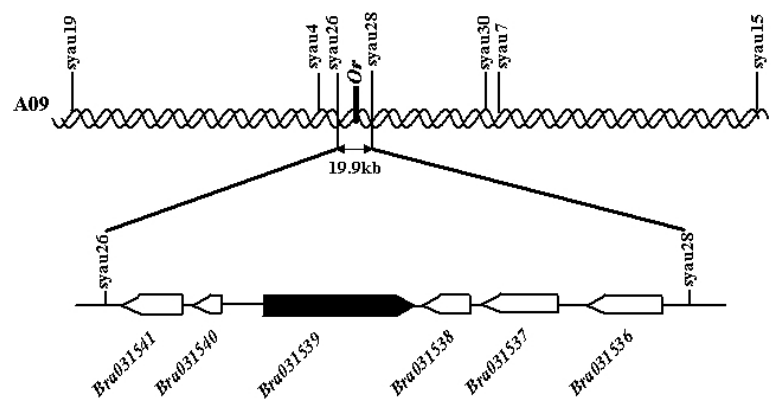

Figure 1. Genetic and physical maps of the or gene locus and candidate gene analysis in Chinese cabbage. Using the molecular markers, syau19 and syau15, or was previously localized to chromosome A09 between KBrH010M08 and KBrB017P15 (Feng et al., 2012), two BAC clones. Using 2,200 F2 homozygous recessive individuals, syau26 and syau 24 , or was fine mapped to a $19.9 \mathrm{~kb}$ region containing six genes, including Bra031539, the predicted candidate gene. 


\section{Cloning of Bra031539 and BLAST analysis of sequences in the two materials}

BLAST analysis of Bra031539 between Chiifu and 07A163 revealed some differences in the nucleic acid and protein sequences between lines, including the following: 88 SNPs were found in the promoter region and two positions contain base deletions, including an 88-bp deletion at the 751-838-bp position and a 7-bp deletion at 937-943 bp (Figure 2a). We identified 13 exons in Bra031539 containing 100 SNPs. A 6-bp deletion was detected in the first exon, which is situated in the 1,477-1,482-bp region in orange head Chinese cabbage line 07A163 (Figure 2b). Early termination of protein translation occurs at the 3' end of Bra031539 in line 07A163 compared to white head Chinese cabbage Chiifu, and the orange head line contains a 252-bp deletion (Figure 2c).

We compared the deduced protein sequences of the two materials, finding that 22 amino acids were altered in the orange head line and 17 amino acids were lost. The 43 SNPs present in the first exon resulted in 15 amino acid changes and the 6-bp deletionled to the loss of two amino acids. The early termination of protein translation occurs due to the 3 '-end DNA deletion in the orange head material (Figure 3).

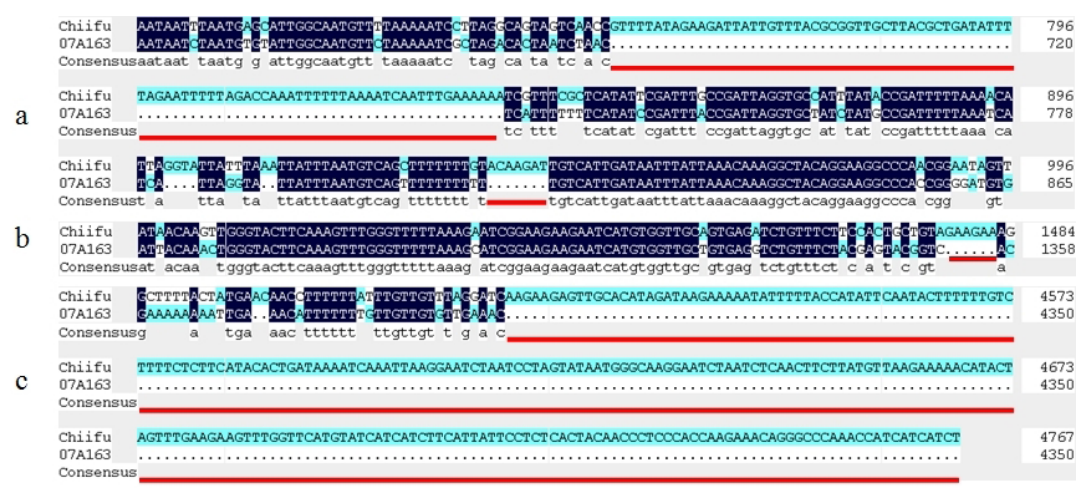

Figure 2. Comparison of Bra031539 nucleic acid sequences in Chiifu and 07A163. a Sequence alignment of the promoter regions between Chiifu and 07A163; red lines indicate 88-bp and 7-bp deletions; b 6-bp deletion in $\mathrm{CDS1}$; c due to early termination at the 3'-end, 252-bp of DNA were deleted in 07A163.

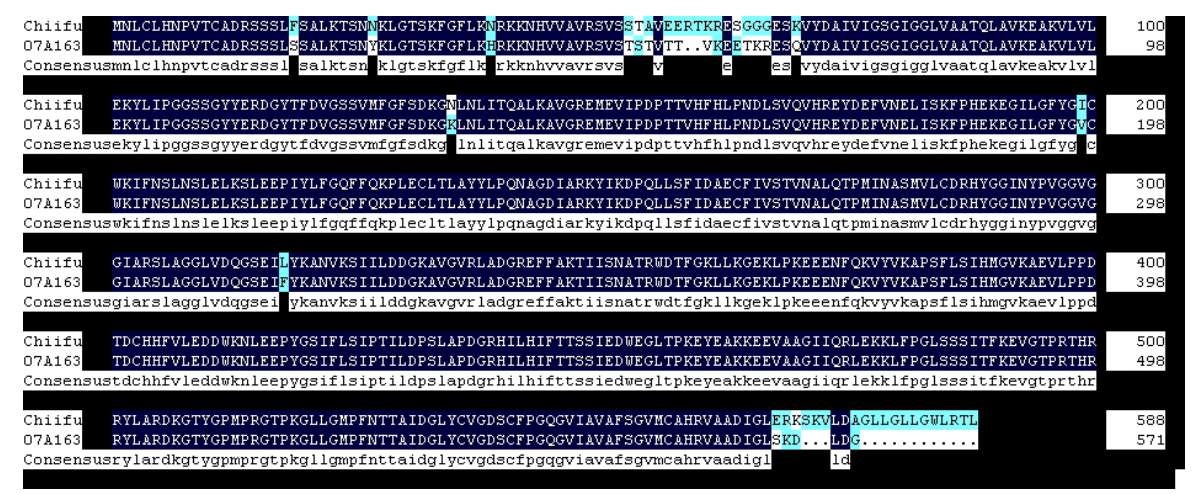

Figure 3. Comparison of Bra031539 protein sequences in Chiifu and 07A163. 22 amino acids were altered in the orange head line and 17 amino acids were lost. The early termination of protein translation occurs due to the 3 '-end DNA deletion in the orange head material. 


\section{Protein sequence analysis of Bra031539}

The full-length sequence of Bra031539 is 3402 bp long. Its 13 coding sequences (CDS) encode 589 amino acid sequences. TargetP analysis showed that Bra031539 is likely a chloroplast transport peptide, while signalP3.0 prediction also revealed that Bra031539 lacks an N-terminal signal peptide.

Protein sequence pattern analysis by Prosite showed that Bra031539 contains 12 caseinkinase II phosphorylation sites, eight protein kinase $C$ phosphorylation sites, one cAMPand cGMP-dependent protein kinase phosphorylation site, $13 \mathrm{~N}$-myristoylation sites, two tyrosine kinase phosphorylation sites, three $\mathrm{N}$-glycosylation sites, and a leucine zipper pattern (Figure 4). SMART analysis revealed that Bra031539 contains four domains: a Pfam:DAO domain, an FAD binding-2 domain, a NAD binding-8 domain and an amino oxidase domain.

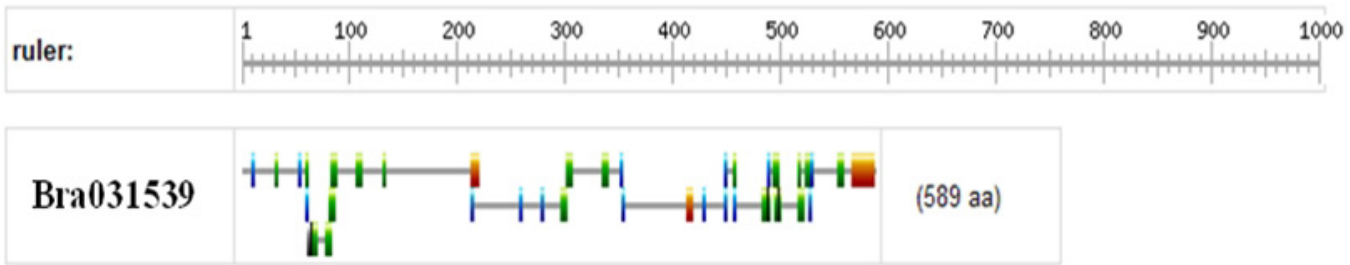

Figure 4. Sites with a high probability of occurrence in Bra031539, as revealed using Scan Prosite. The full-length sequence of Bra031539 is 3,402 bp long encoding 589 amino acid sequences and protein sequence pattern analysis by Prosite showed that Bra031539 contains forty sites.

\section{Development of a molecular marker based on the differences between the two parents}

Three primer pairs were designed based on the promoter region of Bra031539 and were verified through analysis of the white, yellow, and orange Chinese cabbage materials. The results showed that primer pair BR1 amplified different PCR products in the orange head materials than from those of the white and yellow head materials (Figure 5). We performed dominance analysis of all F1 populations (Figure 6). F2 homozygous recessive orange head individuals performed the same as orange head line 07A163. However, in F2 heterozygous individuals, the white head character co-segregated with BR1 (Figure 7). Therefore, the primer pair BR1, which amplifies marker BrProl (Bra031539Promoter1), can be used to distinguish orange head materials from white and yellow head materials.

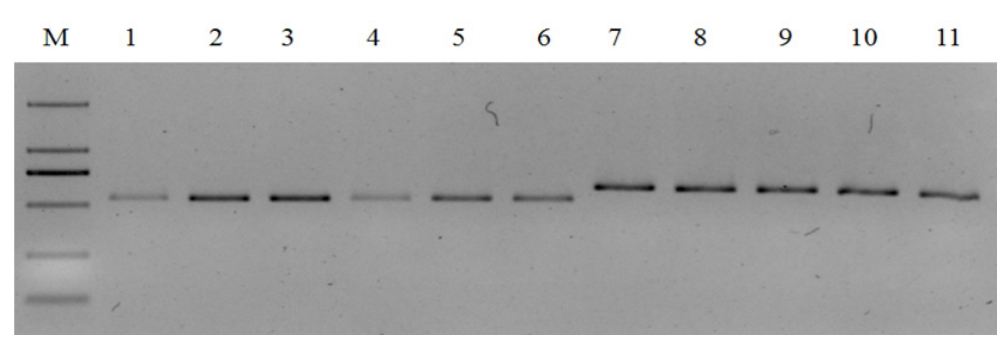

Figure 5. Results of PCR amplification of BrProl in the three test materials. Lane M: DL2000 Markers; lanes 1-6 are orange head Chinese cabbages lines 07A163, Ju65, Ju62, Zhengcai1, Jihong308 and Jinzi-1, respectively; lanes 7 and 8 are white head Chinese cabbage lines Chiifu and Futian50, respectively; lanes 9-11 are yellow head Chinese cabbage lines Jin04-02, Dongbao and 76-F-2, respectively. 


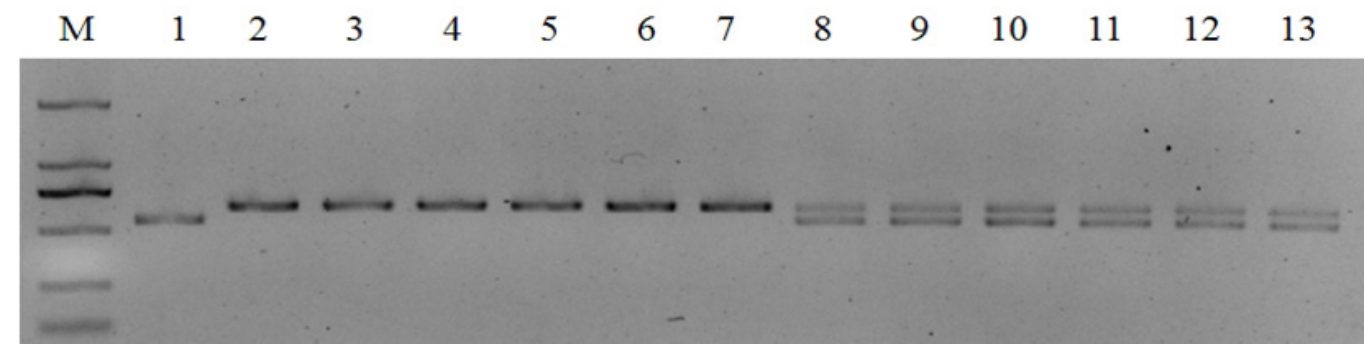

Figure 6. Results of PCR amplification of BrProl in the F1 population. Lane M: DL2000 Markers; lane 1 is orange head Chinese cabbage 07A163; lanes 2-4 are white head Chinese cabbage lines Chiifu, 14A014 and 14A065, respectively; lanes 5-7 are yellow head Chinese cabbage lines 14A036, 14A038 and 14A029, respectively; lanes 8-10 are F1 lines from a cross between white head Chinese cabbage and orange head Chinese cabbage, i.e., Chiifu x 07A163, 14A014 x 07A163 and 14A065 x 07A163, respectively; lanes 11-13 are F1 lines from a cross between yellow head Chinese cabbage and orange head Chinese cabbage, i.e., 14A036 x 07A163, 14A038 x 07A163 and 14A029 x 07A163, respectively.

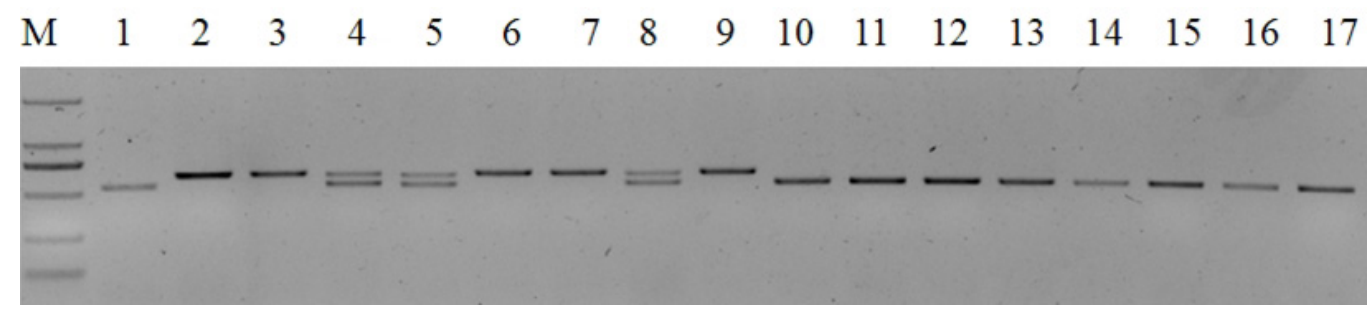

Figure 7. Results of PCR amplification of BrProl in the F2 population. Lane M: DL2000 Markers; lane 1 is orange head Chinese cabbage; lane 2 is white head Chinese cabbage; lanes 3-9 are white head Chinese cabbage in F2; lanes 10-17 are orange head Chinese cabbage in the F2.

\section{DISCUSSION}

Based on the preliminary mapping of the or gene in Chinese cabbage (Feng et al., 2012), we constructed a fine mapping of a population containing 2200 F2 homozygous recessive individuals. Using two SSR markers, syau 26 and syau28, we localized the or gene, which controls the orange head character, to linkage group A09. The physical distance between these two markers is $19.9 \mathrm{~kb}$. Using an F2S4 population, a single recessive gene, $\mathrm{Br}$-or, was previously localized to a $16.7-\mathrm{kb}$ region, with three ORFs (open reading frames) predicted in the target area (Zhang et al., 2013). Br-or was then localized to a $9.47 \mathrm{~kb}$ region containing one predicted gene (Su et al., 2014). Using two insertion-deletion (InDel) markers, SB14001 and SB13049, the target gene was subsequently localized to a $65.555 \mathrm{~kb}$ region containing 17 candidate genes ( $\mathrm{Li}$ et al., 2015). In summary, our results confirm that the target gene that controls the orange head character in Chinese cabbage is located at the end of linkage group A09.

Analysis based on information in the Brassica database (http://brassicadb.org/brad/ index.php) and the National Center for Biotechnology Information (http://blast.ncbi.nlm.nih. gov/Blast.cgi) confirmed the presence of six candidate genes in the target region. Bra031539 encodes the carotenoid isomerase CRTISO, which plays an important role in the carotenoid biosynthetic pathway. CRTISO is the enzyme that coverts tetra-cis-lycopene to all-trans- 
lycopene. The Arabidopsis CRTISO locus was identified through analysis of crtiso mutants exhibiting partial inhibition of lutein biosynthesis in light-grown tissue and the accumulation of poly-cis-carotene precursors in dark-grown tissue (Park et al., 2002). The tangerine mutant in tomato has a deletion in CRTISO and accumulates prolycopene instead of all-translycopene, resulting in orange fruit (Isaacson et al., 2002). The mutation of BrCRTISO1 in Chinese cabbage was predicted to cause the orange-color trait (Lee et al., 2014). Based on the above result, we also predict that Bra031539 is the target gene that controls the orange head character in Chinese cabbage.

Recent studies by several groups have involved sequence alignments between white head Chinese cabbage and orange head Chinese cabbage. Comparison of the OC (orangecolored) and YE (yellow-colored) cultivars revealed that Bra031539 contains two large InDels and many SNPs in the first exon in the OC cultivar. The sequence variations result in 19 amino acid modifications. Lee et al. (2014) proposed that methylation and secondary structures might exist to preclude transcription at the $3^{\prime}$ end of $\mathrm{BrCRTISO1}$ in the OC cultivar. A BAC library was developed to clone BrCRTISO in the orange head material. A 90-bp DNA deletion in the promoter and a 501-bp DNA insertion prior to the stop codon are the distinctive features revealed between the two experimental materials (Su et al., 2014). Li et al. (2015) found that an orange-type A21530 mutant also lacks the 3' end sequence. However, a 141-bp deletion in the promoter region and 6-bp deletion, as well as 53 SNPs in the first exon, were found in orangetype cultivars. Zhang et al. (2015) compared the genomic sequences of two Chinese cabbage materials and found that their differences included an 88-bp deletion in the promoter region, a 501-bp insertion at the 3' end, and two SNPs. The two SNPs cause two amino acid substitutions and a 501-bp insertion, resulting in the addition of 143 amino acids at the end of BrCRTISO.

In summary, the results obtained with these different cabbage materials vary. Due to the longer sequence of Bra031539, we did not obtain the sequence of the $3^{\prime}$ end of orange head Chinese cabbage line 07A163 by gene amplification and clone sequencing. The amplified fragment and sequence assembly were used to obtain the full-length Bra031539 in white head Chinese cabbage Chiifu and orange head line 07A163. Based on BLAST analysis by DNAMAN, two deletions of 88 and $7 \mathrm{bp}$ were detected in the promoter region of Bra031539 in 07A163, which also contains 88 SNPs. A deletion of 6 bp in the first exon results in the failed translation of two amino acids, and 43 of the SNPs lead to 15 amino acid substitutions. The nucleic acid sequence at the $3^{\prime}$ end exhibits frameshift mutations in 07A163 that result in premature termination of protein translation. This might explain why the isomerase activity of CRTISO was lost in this orange head line. Some of our results differ from those of previous studies. Future studies will focus on identifying which mutation results in the inactivation of CRTISO in different orange head cabbage lines.

We designed gene-specific markers based on the nucleotide deletion in the promoter region and verified them in white, yellow, and orange parental lines and in the F1 and F2 generations. Marker BrProl was codominant in the F1 generation and co-segregated in the F2 generation. This marker can therefore be used to distinguish orange materials from white and yellow materials. The use of this marker may enable identification of hybrid orange head materials in the early stage of breeding, which could speed up the breeding process of orange head Chinese cabbage. Compared with the markers developed in fine mapping studies, such as those closely linked to or (Zhang et al., 2008; Feng et al., 2012; Zhang et al., 2013; Su et al., 2014), functional markers are developed in mutated genes, thereby allowing genotypes to be distinguished accurately. At the same time, an allele-specific marker (Br-Pro-Indel) was also 
developed to identify allelic variations in Chinese cabbage cultivars (Zhang et al., 2015). In the current study, six different orange lines, six different yellow lines, four different white lines, and F1 and F2 generation hybrids were used to verify BrPro1. This marker can distinguish orange head Chinese cabbage from yellow and white in both homozygous from heterozygous plants, suggesting that it will be used extensively for marker-assisted selection breeding.

\section{Conflicts of interest}

The authors declare no conflict of interest.

\section{ACKNOWLEDGMENT}

Research supported by the earmarked fund for China Agriculture Research System (\#CARS-25-A-03), the National Natural Science Foundation of China (\#31272157) and Cultivation Plan for Youth Agricultural Science and Technology Innovative Talents of Liaoning Province (\#2014031).

\section{REFERENCES}

Bramley P, Teulieres C, Blain I, Bird C, et al. (1992). Biochemical characterization of transgenic tomato plants in which carotenoid synthesis has been inhibited through the expression of antisense RNA to pTOM5. Plant J. 2: 343-349. http://dx.doi.org/10.1111/j.1365-313X.1992.00343.x

Chen PF (2008). The study of identify and accumulation mechanisms and regulation control of carotenoid on Chinese cabbage. Dissertation, College of Horticulture and Protection, Yangzhou University, China

Chen Y, Li F and Wurtzel ET (2010). Isolation and characterization of the Z-ISO gene encoding a missing component of carotenoid biosynthesis in plants. Plant Physiol. 153: 66-79. http://dx.doi.org/10.1104/pp.110.153916

Cunningham FX, Jr., Chamovitz D, Misawa N, Gantt E, et al. (1993). Cloning and functional expression in Escherichia coli of a cyanobacterial gene for lycopene cyclase, the enzyme that catalyzes the biosynthesis of $\beta$-carotene. FEBS Lett. 328: 130-138. http://dx.doi.org/10.1016/0014-5793(93)80980-9

Cunningham FX, Jr., Pogson B, Sun Z, McDonald KA, et al. (1996). Functional analysis of the beta and epsilon lycopene cyclase enzymes of Arabidopsis reveals a mechanism for control of cyclic carotenoid formation. Plant Cell 8: 1613-1626.

Feng H, Li YF, Liu ZY and Liu J (2012). Mapping of or, a gene conferring orange color on the inner leaf of the Chinese cabbage (Brassica rapa L. ssp. pekinensis). Mol. Breed. 29: 235-244. http://dx.doi.org/10.1007/s11032-010-9542-x

Fraser PD, Truesdale MR, Bird CR, Schuch W, et al. (1994). Carotenoid biosynthesis during tomato fruit development. Plant Physiol. 105: 405-413.

Isaacson T, Ronen G, Zamir D and Hirschberg J (2002). Cloning of tangerine from tomato reveals a carotenoid isomerase essential for the production of $\beta$-carotene and xanthophylls in plants. Plant Cell 14: 333-342. http://dx.doi. org/10.1105/tpc.010303

Lee S, Lee SC, Byun DH, Lee DY, et al. (2014). Association of molecular markers derived from the BrCRTISO1 gene with prolycopene-enriched orange-colored leaves in Brassica rapa [corrected]. Theor. Appl. Genet. 127: 179-191. http://dx.doi.org/10.1007/s00122-013-2209-3

Li F, Murillo C and Wurtzel ET (2007). Maize $Y 9$ encodes a product essential for 15-cis-zeta-carotene isomerization. Plant Physiol. 144: 1181-1189. http://dx.doi.org/10.1104/pp.107.098996

Li PR, Zhang SJ, Zhang SF, Li F, et al. (2015). Carotenoid identification and molecular analysis of carotenoid isomeraseencoding BrCRTISO, the candidate gene for inner leaf orange coloration in Chinese cabbage. Mol. Breed. 35: 1-12. http://dx.doi.org/10.1007/s11032-015-0202-z

Murray MG and Thompson WF (1980). Rapid isolation of high molecular weight plant DNA. Nucleic Acids Res. 8: 43214325. http://dx.doi.org/10.1093/nar/8.19.4321

Nisar N, Li L, Lu S, Khin NC, et al. (2015). Carotenoid metabolism in plants. Mol. Plant 8: 68-82. http://dx.doi. org/10.1016/j.molp.2014.12.007

Park H, Kreunen SS, Cuttriss AJ, DellaPenna D, et al. (2002). Identification of the carotenoid isomerase provides insight into carotenoid biosynthesis, prolamellar body formation, and photomorphogenesis. Plant Cell 14: 321-332. http:// dx.doi.org/10.1105/tpc. 010302 
Pecker I, Gabbay R, Cunningham FX, Jr. and Hirschberg J (1996). Cloning and characterization of the cDNA for lycopene $\beta$-cyclase from tomato reveals decrease in its expression during fruit ripening. Plant Mol. Biol. 30: 807-819. http:// dx.doi.org/10.1007/BF00019013

Qing X and Cheng JZ (2000). The chemical properties and physiological function of lycopene. Food Ferment Ind. 26: 75-82.

Ronen G, Cohen M, Zamir D and Hirschberg J (1999). Regulation of carotenoid biosynthesis during tomato fruit development: expression of the gene for lycopene epsilon-cyclase is down-regulated during ripening and is elevated in the mutant Delta. Plant J. 17: 341-351. http://dx.doi.org/10.1046/j.1365-313X.1999.00381.x

Ruiz-Sola MA and Rodríguez-Concepción M (2012). Carotenoid biosynthesis in Arabidopsis: a colorful pathway. Arabidopsis Book 10: e0158. http://dx.doi.org/10.1199/tab.0158

Su TB, Yu SC, Wang J, Zhang FL, et al. (2014). Loss of function of the carotenoid isomerase gene BrCRTISO confers orange color to the inner leaves of Chinese cabbage (Brassica rapa L. ssp. pekinensis). Plant Mol. Biol. Rep. 33: 648-659. http://dx.doi.org/10.1007/s11105-014-0779-0

Tuan PA, Kim JK, Park NI, Lee SY, et al. (2011). Carotenoid content and expression of phytoene synthase and phytoene desaturase genes in bitter melon (Momordica charantia). Food Chem. 126: 1686-1692. http://dx.doi.org/10.1016/j. foodchem.2010.12.058

Watanabe M, Musumi K and Ayugase J (2011). Carotenoid pigment composition, polyphenol content, and antioxidant activities of extracts from orange-colored Chinese cabbage. LWT Food Sci. Technol. 44: 1971-1975. http://dx.doi. org/10.1016/j.lwt.2011.04.010

Zhang FL, Wang GC, Wang M, Liu XC, et al. (2008). Identification of SCAR markers linked to $o r$, a gene inducing betacarotene accumulation in Chinese cabbage. Euphytica 164: 463-471. http://dx.doi.org/10.1007/s10681-008-9721-6

Zhang J, Yuan H, Fei Z, Pogson BJ, et al. (2015). Molecular characterization and transcriptome analysis of orange head Chinese cabbage (Brassica rapa L. ssp. pekinensis). Planta 241: 1381-1394. http://dx.doi.org/10.1007/s00425-015$\underline{2262-Z}$

Zhang JC, Zhou WJ, Xu Q, Tao NG, et al. (2013). Two lycopene $\beta$-cyclases genes from sweet orange (Citrus sinensis L. Osbeck) encode enzymes with different functional efficiency during the conversion of lycopene-to-provitamin A. $J$. Integr. Agric. 12: 1731-1747. http://dx.doi.org/10.1016/S2095-3119(13)60366-4 